\title{
The Advanced Energetic Pair Telescope for gamma-ray polarimetry
}

\author{
Stanley D. Hunter ${ }^{1}$ \\ NASA/Goddard Space Flight Center, Greenbelt, MD 20771
}

\begin{abstract}
The Advanced Energetic Pair Telescope (AdEPT), a future NASA/GSFC MIDEX mission, is being developed to perform high-sensitivity medium-energy $(5-200 \mathrm{MeV})$ astronomy and revolutionary gamma-ray polarization measurements. The enabling technology for AdEPT is the ThreeDimensional Track Imager (3-DTI), a large volume gaseous time projection chamber with 2dimentional micro-well detector (MWD) readout. The low density and high spatial resolution of the 3-DTI allows AdEPT to achieve high angular resolution $\left(\sim 0.5^{\circ}\right.$ at $\left.67.5 \mathrm{MeV}\right)$ and, for the first time, exceptional gamma-ray polarization sensitivity. These capabilities enable a wide range of scientific discovery potential for AdEPT. The key science goals of the AdEPT mission include: 1) Explore fundamental processes of particle acceleration in active astrophysical objects, 2) Reveal the magnetic field configuration of the most energetic accelerators in the Universe, 3) Explore the origins and acceleration of cosmic rays and the Galactic MeV diffuse emission, 4) Search for dark matter in the Galactic center, and 5) Test relativity with polarization measurements. We report on the latest developments of the MWDs for the 3-DTI.
\end{abstract}

Keywords: Gamma ray, polarimetry, medium-energy, instrument, time projection chamber, microwell detector

\section{INTRODUCTION}

The currently operating Fermi /Large Area Telescope (LAT) and the AGILE missions have made important advances in the high-energy gamma-ray range, above $\sim 200 \mathrm{MeV}$. By comparison, the medium energy gamma-ray range is relatively unexplored, having been initially studied with only limited sensitivity by CGRO/COMPTEL (1-30 MeV) and CGRO/EGRET (30 MeV-30 GeV). A future gamma-ray telescope, optimized to detect medium energy gamma-ray spectra and polarization below $\sim 200 \mathrm{MeV}$ energy via pair-production will open a new window on the high energy universe and would be a natural successor to the Fermi Mission. This telescope should provide adequate sensitivity and enough angular resolution in order to make detailed observations with high resolution and polarization sensitivity in the energy range from a few hundred $\mathrm{keV}$ to several hundred $\mathrm{MeV}$. Such an instrument will enable the study a wide range of astrophysical phenomena with a new capability. Medium energy observations are required in order to fully understand phenomena such as pulsars, supernova remnants, diffuse emission, blazars and other active galactic nuclei.

The first measurements of the radio polarization of the Sun were published in the mid 1940s and followed by detection from the Crab nebula in 1957 and other sources. Optical polarization effects

\footnotetext{
${ }^{1}$ Code 661, Stanley.d.hunter@nasa.gov
} 
were observed much earlier. Allegedly the Vikings used skylight polarization for navigation, but the first recorded documentation was by a Danish physicist, physician and mathematician, Erasmus Bartholinus (1625-1698) ${ }^{[1]}$, who described the double refraction of calc-spar (or Iceland spar, a variety of calcite). It was Christian Huygens (1629-1695), a Dutch physicist and astronomer who made the fundamental discovery of polarization in $1690^{[2]}$. Measurement of polarization at microwave, infrared, and ultraviolet frequencies have followed. The first X-ray polarization measurement of the Crab nebula at $2.6 \mathrm{keV}$ was made by Novick ${ }^{[3]}$. This work has led to the Imaging X-ray Polarimetry Explorer (IXPE) instrument (2-8 keV) which will be launched in $2021^{[4]}$. Observations of Cygnus X-1 with INTEGRAL, launched in 2002, have shown significant polarization in the $0.4-2 \mathrm{MeV}$ energy range. NuSTAR, launched in 2012, has polarization sensitivity over the $\sim 3-80 \mathrm{keV}$ energy range ${ }^{[5]}$.

Polarization is a fundamental property of electromagnetic radiation that can be measurable in photoelectric, Compton scattering, and pair interactions ${ }^{[6]}$. The intensity of electromagnetic radiation (radio, infrared, light, or X-ray) gives us primary information about the distribution of the baryonic matter in the Universe, the measurement of polarization enables us to investigate many details of the structure of the magnetic fields responsible for the particle acceleration.

Polarization can be measured for photons via photo-electric, Compton scattering, or pair production interactions. The angular distribution of the secondary particles are azimuthally asymmetric. For an experiment that measures the azimuthal angle of the secondary particle plane the cross-section for $100 \%$ linear polarized photons can be written ${ }^{[6]}$.

$$
d \sigma=a\left(1+\lambda \cos ^{2} \varphi\right) d \varphi / 2 \pi
$$

Where $\lambda$, the energy dependent functions for on the interaction processes, is shown in Figure $1^{[7]}$. The value of $\lambda$ for pair production increases rapidly from 0 at $E_{\gamma} / 2 m_{e}=$ 1 , reaches a maximum value of $\sim 0.54$ at $E_{\gamma} / 2 m_{e}=2$ and then decreases slowly to an asymptotic value of $\sim 0.3$.

We note that for astrophysical sources with power-law spectra, the value $\lambda$ for pair production is $\sim 1.5$ times that for Compton scattering above $E_{\gamma} / 2 m_{e} \approx 2$ and, in the case of pair production, $\lambda$ is not sensitive to the interaction material ${ }^{[6]}$.

For this reason, we have concentrated our efforts on developing a pair production polarimeter and report on the development of the Advanced Energetic Pair Telescope (AdEPT), a medium-energy gamma-ray polarimeter. The key technology for AdEPT is the Three-Dimensional Track Imager (3-DTI) which combines a gaseous time projection chamber with the negative ion drift technique ${ }^{[8]}$ to provide the low density, large volume, high spatial resolution needed for gamma ray polarization ${ }^{[9]}$. The characteristics of the AdEPT instrument and its capabilities are described by Hunter et al. ${ }^{[10]}$. We describe the recent advances being

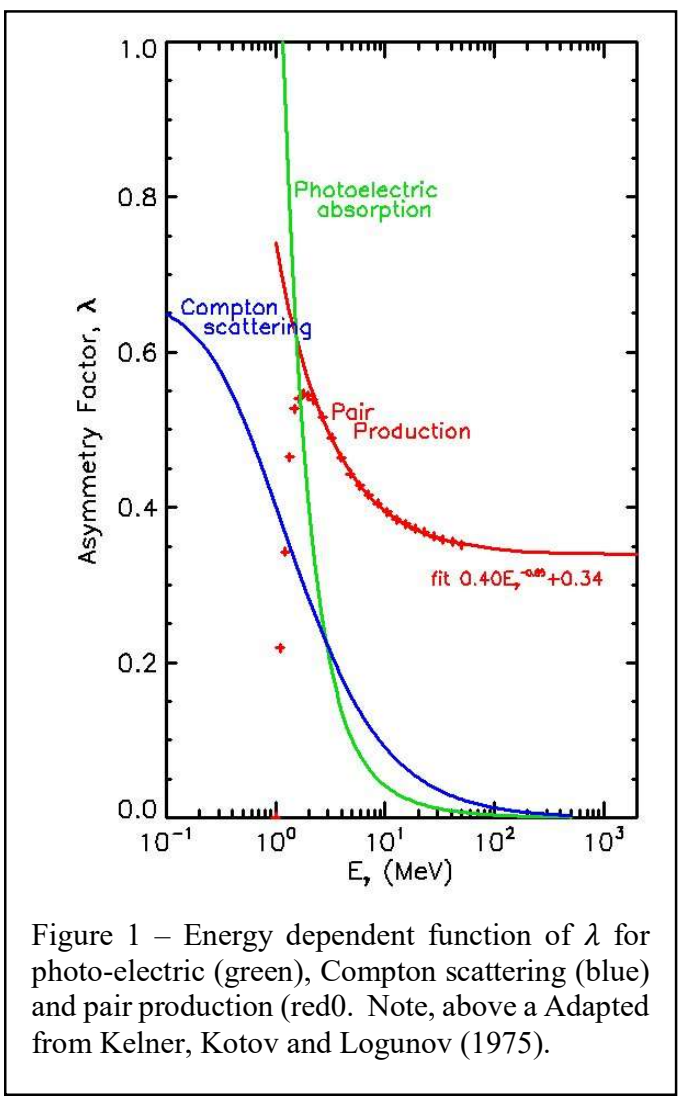


made in the 2-D micro-well detector (MWD) readout for the 3-DTI technology.

\subsection{Scientific importance of polarization, an example}

The scientific advantages of polarization measurements are well demonstrated by the recent INTEGRAL observations of Cygnus $\mathrm{X}-1^{[11]}$. These results, combined with BeoboSax, Fermi-LAT, Magic, and CTA estimates, by Zanin, et al. ${ }^{[12]}$ are shown in Figure 3. The INTEGRAL results indicate two components, the first component, dominant below $3 \times 10^{5} \mathrm{eV}$, shows weak or no polarization whereas the second component, proposed to originate from the jet and dominant above $3 \times 10^{5} \mathrm{eV}$, shows strong polarization. These two components clearly originate in two separate regions of Cygnus $\mathrm{X}-1$. The

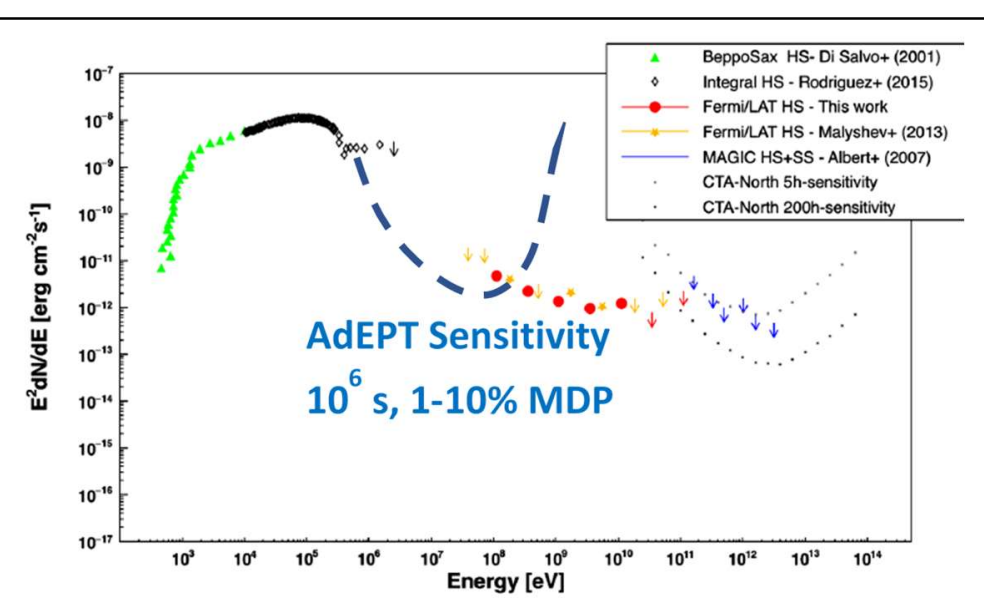

Figure 3 - Spectral energy distribution of Cygnus X-1 from X-rays up to $\mathrm{TeV}$ energies when in the HS. The dashed blue line indicates the AdEPT sensitivity in $106 \mathrm{~s}$. Over this energy range the AdEPT MDP varies from about 1 to $10 \%$. Adapted from [12].
AdEPT sensitivity, shown as the dashed blue line in Figure 3 covering the energy range from a few $\mathrm{MeV}$ up to $100 \mathrm{~s}$ of $\mathrm{MeV}$, will bridge the energy range between INTEGRAL and Fermi and determine if the second polarized component extends to Fermi energy or cuts off at a few $\mathrm{MeV}$ indicating that the Fermi emission has another explanation.

\section{THREE-DIMENSIONAL TRACK IMAGER}

AdEPT will explore the physical structure of the magnetic fields that drive the most powerful accelerators in the universe by measuring, for the first time, the polarization of medium-energy gamma rays. The enabling technology for AdEPT is the Three-Dimensional Track Imager (3DTI), shown schematically in Figure 2. The 3-DTI combines a large volume gaseous Time Projection Chamber (TPC) with a two-dimensional Micro-Well Detector (MWD) readout plane. The 3-DTI, operation described below, enables AdEPT to achieve the high gamma-ray polarization sensitivity and kinematically limited angular resolution needed to realize the science goals.

In operation gamma rays interact with the AdEPT/3-DTI detector gas $\left(95 \% \mathrm{Ar}+5 \% \mathrm{CS}_{2}\right)$ in the $\mathrm{TPC}$ volume producing an electron and positron pair that traverse the

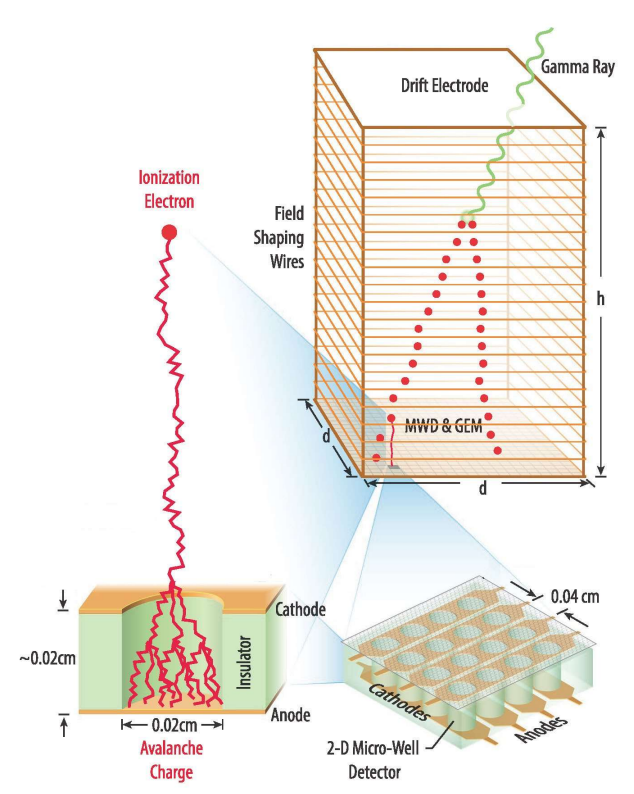

Figure 2 - (upper right) Schematic diagram of the Three-Dimensional Track Imager (3-DTI) electrode structure. A gamma ray converts into an electron-positron pair, the par electrons traverse the gas volume leaving a trail of ionization electrons (red dots). The ionization electrons drift towards the micro-well detector (MWD) at the bottom of the 3-DTI volume (lower right) and the ionization electrons (left) drift into MWD wells producing a Townsend avalanche. 
gas and lose energy by ionizing gas atoms along their paths. These ionization electrons, if allowed to drift freely through the gas would suffer substantial diffusion ( 300-400 $\mu \mathrm{m} / \sqrt{\mathrm{cm}}$ ), exceeding the detector pitch after drifting only moderate distances (few $\mathrm{cm}$ ). The diffusion is dramatically reduced $(\sim 70-80 \mu \mathrm{m} / \sqrt{\mathrm{cm}})$ by adding an electronegative component (carbon disulfide $\mathrm{CS}_{2}$ ) to the gas. Each ionization electron forms a negative ion $\left(\mathrm{CS}_{2}+\mathrm{e}^{-} \rightarrow \mathrm{CS}_{2}^{-}\right)$ that drifts in a uniform $(\sim 1 \mathrm{kV} / \mathrm{cm})$ electric field towards the readout and into one of the micro-wells. Upon reaching the strong electric field within the micro-wells $(\sim 100$ $\mathrm{kV} / \mathrm{cm}$ ), shown schematically in the lower right of Figure 2 and in more detail in Figure 4, the ionization electron is knocked free of the negative ion and accelerated producing

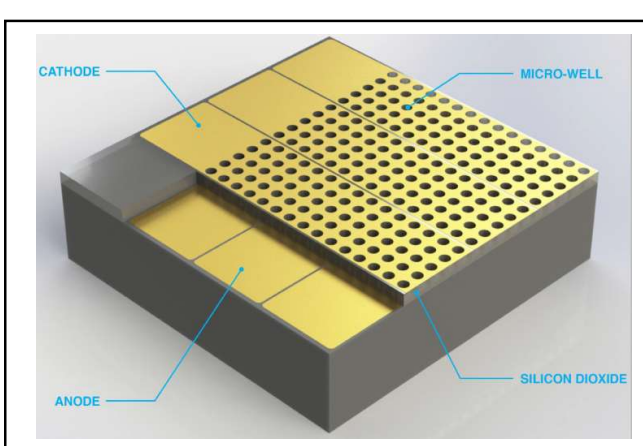

Figure 4 - Schematic drawing of the advanced MWD. The reduced diameter of the advanced MWD micro-wells allows for multiple microwells per pixel.

a Townsend avalanche. The avalanche charge is collected on the anode (X-coordinate) of the MWD and simultaneously an image charge is collected on the MWD cathode (Y-coordinate). The Zcoordinate of each ionization electron is determined from the relative time of arrival. The advantage of using negative ion drift over free electron drift is that diffusion, "blurring," of the charged-particle tracks over equal drift distances is significantly reduced ( 15-30 times lower).

The collision process which frees the electrons from the negative ions results in a two order of magnitude reduction in the avalanche gain of the MWD. Achieving the AdEPT science goals requires that individual ionization electrons (negative ions) be detected by the TPC/MWD readout. Increasing the gas gain of our laser machined polyimide MWDs by a factor of $100-200$, to $\sim 5 \times 10^{5}$, is not viable and motivated our design of the advanced MWD technology. We expect to achieve negative ion gas gain approaching $10^{5}$ with stable operation and substantially reduced breakdown probability.

\section{FABRICATION OF THE ADVANCED MWD}

The technique developed at GSFC fabricate MWD utilized a UV excimer laser to micro-machine the wells in a commercially patterned polyimide flex circuit substrate ${ }^{[13]}$. The orthogonal MWD electrodes etched on the top and bottom surfaces of the polyimide flex-circuit defined the $200 \mu \mathrm{m}$ diameter micro-wells on $400 \mu \mathrm{m}$ center-to-center spacing. The polyimide MWDs worked very well with gas gains in excess of $10^{4}$. Large area, $30 \times 30 \mathrm{~cm}^{2}$, MWDs were used to demonstrate neutron imaging via proton scattering ${ }^{[14]}$. The lifetime of these MWDs was found, however, to be limited to a few months. The ability of the MWDs to support the $100 \mathrm{kV} / \mathrm{cm}$ electric field in the wells decreased with time and the MWD hold-off voltage decreased to the point where the gas gain decreased to less than $10^{2}$. This irreversible failure was attributed to charge migration in the polyimide. Optimizing the MWD performance continues to be a key aspect of the AdEPT/3-DTI mission development.

In 2015, we started a program, working with three companies, to fabricate MWDs taking advantage of the high resistivity of glass (silicon dioxide) as the MWD insulator. Working with these companies, we were able to pattern the MWD electrodes on 100-150 mm diameter glass wafers and etch the wells through the glass. We were also able to reduce the diameter of the wells, pattern electrodes with $100 \mu \mathrm{m}$, center-to-center spacing, and to fabricate $8.5 \times 8.5 \mathrm{~cm}^{2} \mathrm{MWDs}$. The large area readout plane for the AdEPT mission will be assembled by tiling together an array of these individual tiles with anodes and cathodes electrically connected together. 


\section{ADVANCED MWD FABRICATION}

We have pursued the advanced MWD fabrication with three separate companies, each offering a different technique. The current status of these approaches, and future objectives, being explored with these companies are described below.

The PlanOptik technology starts with an array of $50 \mu \mathrm{m}$ square pillars etched into a silicon wafer. This array of pillars corresponding to the micro-wells is then covered with molten glass and the wafer is thinned down to expose the silicon structures embedded in the glass. The wafers are returned to GSFC where the MWD cathode electrodes

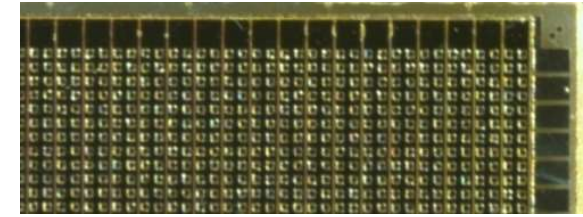

Figure 5 - Corner of PlanOptik MWD. Columns of individual wells $\sim 50 \mu \mathrm{m}$ square are visible. Each cathode electrode, $200 \mu \mathrm{m}$ wide, consists of two columns of wells and terminates in a bond pad at top. Bond pads for the lower level of anode electrodes are visible at the right.

are patterned on the glass/silicon wafer. The anode electrodes are deposited onto another glass wafer used as a carrier. The two glass wafers are thermal-compression bonded at GSFC and the silicon features are etched out at PlanOptik leaving the micro-well structures shown in Figure 5.

The PlanOptik fabrication encountered alignment issues caused by motion of the molten glass. The first detectors, received in 2017, had residual silicon on the well walls resulting in low resistivity, $\sim \mathrm{M} \Omega$, between the cathode and anode electrodes. This problem was corrected by PlanOptik and MWDs were fabricated with $G \Omega$ resistance between the electrodes. Fabrication of these detectors will be completed about June 2018.

The 5microns (Germany) technology provides nickel plating and anisotropic etching of Photo-Sensitive-GlassCeramic (PSGC). The PSGC, when exposed to UV radiation, changes its structure into a ceramic form that has much higher etch rate than the original glass structure. These two process steps, not available in DDL, will allow for a nearly complete internal fabrication of MWDs by the DDL. We expect to complete this MWD development in June 2018.

The 3-D Glass Solutions (Albuquerque) technology is also based on PSGC fabrication of MWDs. This company fabricated MWDs with $100 \mu \mathrm{m}$ diameter wells and $200 \mu \mathrm{m}$ wide electrodes, shown in Figure 6 were received in January 2018. Even though the electrode structure of these

3D Glass MWD

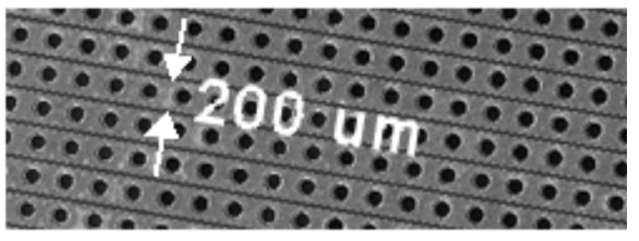

Figure 6 - Portion of 3D Glass MWD showing individual $200 \mu \mathrm{m}$ wide cathode electrodes. The micro-well diameter is $\sim 100 \mu \mathrm{m}$.

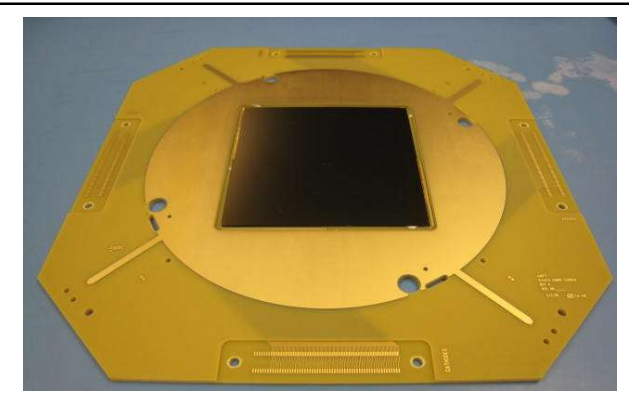

Figure 7 - a 3-D Glass MWD assembly with the detector (center) mounted on a PCB. Wire bonds connect the MWD electrodes to the connector pads around the periphery of the PCB. The circular gold plated electrode provides uniformity of the electric field from the MWD cathode to the base of the cylindrical drift grid, not shown for clarity.

MWDs was not correct (the anode electrodes, on the bottom surface of the tile, were not accessible from the top of the MWD, as indicated in Figure 4, they were mounted on test PCBs, shown in Figure 7 and tested using ${ }^{241} \mathrm{Am}$, an alpha source, in P-10 gas (90\% argon, 10\% methane, at $1 \mathrm{~atm}$ ), a common 
proportional chamber gas, in our test chamber shown in Figure 8. These initial tests confirmed the correct structure of the anode and cathode electrodes and that they were exposed to the gas. Additional tests using ${ }^{55} \mathrm{Fe}$, an X-ray source, were done to determine the MWD gain. These results are shown in Figure 9. The measured gas gain was low, but higher gain was not expected due to the $85 \mu \mathrm{m}$ depth of the micro-wells or possible defects in the metallization around the wells. We are working with 3-D Glass Solutions to correct the electrode structure and to increase the depth of the wells.

\section{FUTURE PLANS}

We expect all three companies to deliver testable MWDs in the next few months. These MWDs will be tested and the fabrication iterated if necessary. We will then place an order or $\sim 40$ tiles and assemble them into a $\sim 50 \times 50 \mathrm{~cm}^{2}$ readout array. A separate research program at GSFC is exploring the use aerosol jet printing to interconnect the anodes and cathodes of the MWD array to each other and to the front-end electronics Paquette. We plan to assemble a prototype 3-DTI model and a complete AdEPT prototype within two years.

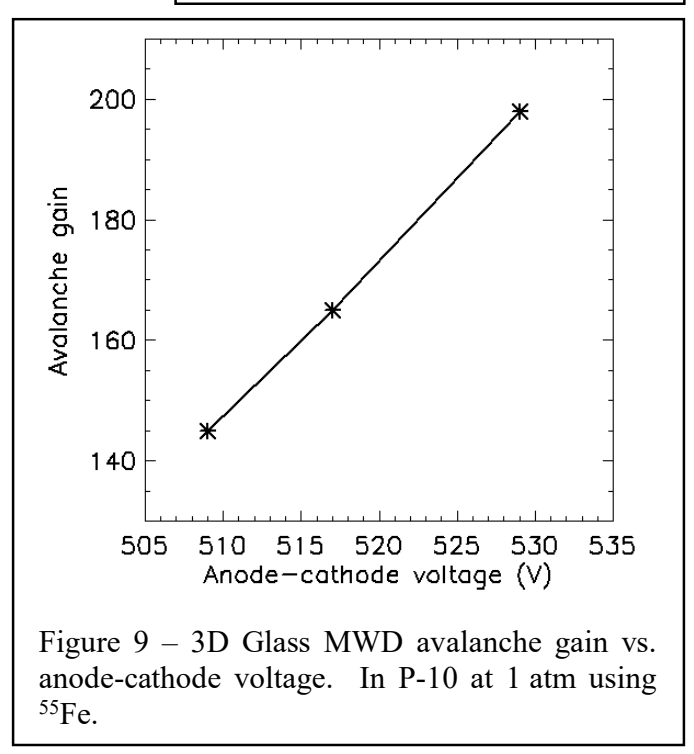

\section{REFERENCES}

\section{[1] https://en.wikipedia.org/wiki/Rasmus_Bartholin}

[2] Horvath, G., https://arago.elte.hu/sites/default/files/DSc-Thesis-2003-GaborHorvath-01.pdf, (2003)

[3] Novick, R., et al., "Detection of X-Ray Polarization of the Crab Nebula," Astrophys. J. 1972, 174, L1 (1972)

[4] https://ixpe.msfc.nasa.gov/ Deininger, W., "IXPE: Imaging X-Ray Polarimetry Explorer Mission," IEEE Aerospace Conference, Yellowstone Conference Center, Big Sky, MT, USA, (2017)

[5] Lotti, S., et al., "Polarization studies with NuSTAR," (2012)

https://arxiv.org/ftp/arxiv/papers/1207/1207.2063.pdf 
[6] Kotov, Yu. D. "Methods of Measuring Gamma-Ray Polarization," Space Sci Rev 49, 185-95 (1988)

[7] Kel'ner, Kotov, and Logunov, "Methods of measuring linear polarization of gamma quanta," Sov. J. Nucl. Phys. 21(3), 313-5 (1975)

[8] Martoff, C. J., et al., "Negative ion drift and diffusion in a TPC near 1 bar," Nucl. Instr. Meth. A $555,55-58(2005)$

[9] Bernard, D., "Polarimetry of cosmic gamma-ray sources above e+ e- pair creation threshold," Nucl. Instr. Meth. A 729, 765-780 (2013)

[10] Hunter, S. D., et al., "A pair production telescope for medium-energy gamma-ray polarimetry," Astroparticle Physics 59, 18-28 (2014)

[11] Rodriguez, J., et al., "Spectral State Dependence of the 0.4-2 MeV Polarized Emission in Cygnus X-1 seen with INTEGRAL/IBIS, and Links with the AMI Radio Data," ApJ, 807, 17 (2015)

[12] Zanin, R., "Gamma rays detected from Cygnus X-1 with likely jet origin,” A\&A, 596 A55 (2016)

[13] Deines-Jones, P., et al., "Imaging micro-well proportional counters fabricated with masked UV laser ablation," Nucl. Inst. Meth. A 477, 55-58, (2002); Deines-Jones, P., et al., "Large-area imaging micro-well detectors for high-energy astrophysics," Nucl. Inst. Meth. A 478, 130-134, (2002)

[14] Son, S. et al., "Neutron Imaging Camera," IEEE Conf. 978-1-4244-9105-6, 298-301 (2010)

[15] Paquette, B., "Direct Write Printing on Thin and Flexible Substrates for Space Applications," Additive Manufacturing for Defense and Aerospace, Arlington, VA (2016) 